\title{
Conservation of species interaction networks
}

\author{
Jason M. Tylianakis ${ }^{\mathrm{a}, *}$, Etienne Laliberté ${ }^{\mathrm{b}}$, Anders Nielsen ${ }^{\mathrm{c}}$, Jordi Bascompte ${ }^{\mathrm{d}}$ \\ ${ }^{\text {a }}$ School of Biological Sciences, University of Canterbury, Private Bag 4800, Christchurch, New Zealand \\ ${ }^{\mathrm{b}}$ School of Forestry, University of Canterbury, Private Bag 4800, Christchurch 8140, New Zealand \\ ${ }^{c}$ Centre for Ecological and Evolutionary Synthesis (CEES), Department of Biology, University of Oslo, P.O. Box 1066 Blindern, 0316 Oslo, Norway \\ d Integrative Ecology Group, Estación Biológica de Doñana, Calle Américo Vespucio s/n, 41092 Sevilla, Spain
}

\section{a b s t r a c t}

Keywords: Food

web Pollination

Global change

Invasion

Climate change

Global warming

Land use change

Parasitoid

Mutualism

\begin{abstract}
Recent work has shown that antagonist (e.g. predator-prey food web) and mutualist (e.g. pollinatorplant) network structure can be altered by global environmental change drivers, and that these alterations may have important ecosystem-level consequences. This has prompted calls for the conservation of network structure, but precisely which attributes of webs should be conserved remains unclear. Further, the extent to which network metrics characterise the spatiotemporally-variable dynamic structure of interacting communities is unknown. Here, we summarise the attributes of web structure that are predicted to confer stability or increased function to a system, as these may be of greatest interest to conservation biologists. However, empirical evaluation of these effects is lacking in most cases, and we discuss whether stability is even desirable in all contexts. The incorporation of web attributes into conservation monitoring requires that changes in these attributes can be recorded (sampled) with relative ease. We contrast the sensitivity of metrics to sampling effort, and highlight those (such as nestedness and connectance) that could easily be incorporated into conservation monitoring. Despite our growing understanding of the characteristics of food webs that confer stability and function, numerous practical challenges need to be overcome before the goal of conserving species interaction networks can be achieved.
\end{abstract}

\section{Introduction}

All species interact with other species in complex antagonistic (e.g. predator-prey) or mutualistic (e.g. pollinator-plant) networks. Perhaps for simplicity, conservation has so far largely ignored the structure of these networks and has instead tended to deal with threat status on a species by species basis. However, failure to consider the ecological network within which a threatened species is embedded may lead to counterproductive management measures. For example, removal of exotic plants may lead to reduced pollination of a rare native plant through changes in the population of pollinators that feed on both natives and exotics (Carvalheiro et al., 2008). Furthermore, recent studies showing effects of anthropogenic changes on the structure of interaction networks (Albrecht et al., 2007; Lopezaraiza-Mikel et al., 2007; Memmott et al., 2007; Aizen et al., 2008) even when species richness is unaffected (Tylianakis et al., 2007) lend support to previous calls for the conservation of network structure (e.g. Thompson, 1994; McCann, 2007). Despite this potential importance, the specific aspects of network structure that should be prioritised in

\footnotetext{
* Corresponding author. Tel.: +64 33642735 .

E-mail address: jason.tylianakis@canterbury.ac.nz (J.M. Tylianakis).
}

conservation efforts or the amount of structural alteration that networks can tolerate before functional changes become apparent remain unclear (Tylianakis et al., 2008). Here we discuss different aspects of ecological network structure that could be conserved, depending on specific conservation priorities, and the future research required to inform such decisions. We assess the usefulness of various structural network metrics for conservation, and subsequently discuss some of the practical challenges that must be overcome before we can even begin to consider conserving entire interaction networks.

Obviously, conserving the species that form interaction networks is a pre-requisite for conserving the networks themselves. However, networks are more than the sum of their component parts (Lewinsohn et al., 2006; Montoya et al., 2006; Pascual and Dunne, 2006; Bascompte and Jordano, 2007). For interactions between species to occur, the species must not only be present, but also co-occur in space and time. This co-occurrence can be affected by global environmental changes (Memmott et al., 2007; Hegland et al., 2009; Laliberté and Tylianakis, in press), altering the interaction structure of a web beyond that which would occur through changes in species diversity or abundances alone. Further, focusing strictly on a single group of organisms (e.g. plants) misses the fact that their persistence can be influenced by the presence of other 

groups of species with which they interact (e.g. pollinators; Bascompte et al., 2006; Fontaine et al., 2006). Thus, a strict focus on conserving species diversity alone will not necessarily conserve network structure, yet network structure may contribute to biodiversity maintenance (Bascompte et al., 2006; Bastolla et al., 2009).

Determining conservation priorities is inherently subjective. Current conservation foci include 'flagship' or 'iconic' species, biodiversity (often measured simply as the number of species), rare or threatened species, or threatened habitats (with the implicit assumption that this will result in the conservation of numerous species). Which of these strategies is employed generally depends on social, economic or practical constraints (Wilson et al., 2007). Similarly, with regard to the conservation of species interaction networks, we must be specific about which attributes should be conserved. Given the complexity of species interaction networks (May, 1973; Montoya et al., 2006; Pascual and Dunne, 2006; Bascompte and Jordano, 2007), advocating a single attribute of their structure that deserves priority for conservation would be unwise given the current state of knowledge. Instead, we outline some of the different options, focusing on appropriate measures for quantifying changes in these attributes, and how these changes affect different ecosystem-level properties such as stability and functional rates.

2. Conserving characteristics that promote system stability and functional rates

Decades of theoretical work have suggested that network structure may affect ecosystem stability (May, 1973; McNaughton, 1978; Pimm, 1979; Neutel et al., 2002; Krause et al., 2003; McCann et al., 2005). However, stability is a multi-faceted concept in ecology (Grimm and Wissel, 1997) and defining which particular facets of stability are relevant to a given situation is critical (Ives and Carpenter, 2007). The definition of stability is not merely a question of semantics; rather, it may determine the attributes of the web that should be conserved, and even whether maintaining stability itself is congruent with conservation objectives.

Conservation usually aims to preserve species diversity in general, or certain charismatic, endemic or endangered species. Thus, the usual definitions of stability with regard to network structure concern resistance to secondary extinctions following species loss (e.g. Solé and Montoya, 2001; Terborgh et al., 2001; Dunne et al., 2002a; Memmott et al., 2004; Srinivasan et al., 2007; Rezende et al., 2007), network integrity (not breaking up into parts; Solé and Montoya, 2001; Dunne and Williams, 2009) and resistance to species invasions (Post and Pimm, 1983; Bartomeus et al., 2008). In the former case, species extinction would be a direct result of environmental changes such as habitat loss, and the interaction (link) between species would be lost when one of the partners disappears. However, loss of the link itself due, for example, to rarity or phenological mismatch of the interacting partners (Visser and Both, 2005; Hegland et al., 2009), may also potentially drive extinction of one or both partners. Thus, the mere presence of two interacting species does not guarantee that their interaction will be realised (Laliberté and Tylianakis, in press), and loss of the interaction could potentially also drive secondary species loss when one or more interacting partners depends strongly on the other. In the following sections, we describe in detail how particular structural attributes of species interaction networks influence these two facets of stability.

Recently, conservation science has shifted its focus from strictly preserving species diversity, to also considering the provision of vital ecosystem services and human welfare in general (e.g. Dobson et al., 2006; Armsworth et al., 2007; Kareiva et al., 2007; Tallis et al., 2008). This view recognises that preservation of species diversity is not only desirable for philosophical or moral reasons (Calli- cott et al., 1999), but also because biodiversity loss can actually threaten human well-being (Millennium Ecosystem Assessment, 2005; Díaz et al., 2006). This utilitarian approach to conservation focuses on the composition and diversity of key "service-provider" species (Luck et al., 2009). However, that view acknowledges that seemingly “redundant” species (Walker, 1992) still need preservation, because these species may become key service providers following environmental change (Ehrlich and Walker, 1998; Naeem, 1998). Hence, the type of stability that the functional approach to conservation targets is the stability of provision of key services, but not necessarily stability of species composition. An additional short-term conservation goal is to enhance the rates of key processes and services. We discuss in the following sections which attributes of network structure are relevant to these goals. In particular, we focus on pollination and biological pest control, because these are important services that have received considerable attention in the literature.

\section{Structural attributes of networks}

\subsection{Interaction diversity}

\subsubsection{What is it?}

Interaction diversity is directly analogous to species diversity. In its simplest form (i.e. interaction richness), it is the number of interactions or 'links' within the network. However, just as species diversity can be more than just species richness, interaction diversity is usually measured in terms relative to the number of species in the network (e.g. connectance), rather than just interaction richness.

\subsubsection{Why is it important?}

Greater interaction diversity may increase the rate of ecosystem processes. This has been tested predominantly on highly simplified bipartite networks where one trophic level is either monospecific or contains few species. For example, higher predator diversity led to greater herbivore suppression in a collard-aphid system (Snyder et al., 2006). This was attributed to greater resource exploitation with greater predator diversity (i.e. complementarity effects), though there was also evidence for sampling effects (i.e. greater chance of having efficient predator species under higher diversity) (Snyder et al., 2006). Similarly, in mutualistic pollinator-plant networks higher pollinator diversity increased seed set through higher functional complementarity (Hoehn et al., 2008). Yet despite these positive impacts of interaction richness on ecosystem function, interference between consumer species can sometimes outweigh potential complementarity effects, leading to reduced ecosystem function with increasing consumer diversity (Montoya et al., 2003; Finke and Denno, 2004).

Interaction diversity can also stabilise the rate of ecosystem processes through time under fluctuating environmental conditions. If, for example, a diverse array of predators attacked a particular pest species, compensation between different predator species following environmental change could maintain pest control through time. This process, known as 'the insurance hypothesis' (Yachi and Loreau, 1999), was developed from the earlier concept of ecological redundancy (Walker, 1992; Lawton and Brown, 1993; Naeem, 1998). Ecological redundancy assumes, however, that higher species richness implies higher diversity of behavioral, physiological or overall fitness responses to environmental change (i.e. response diversity), something which should not be taken for granted (Elmqvist et al., 2003). Response diversity is critical in maintaining ecosystem resilience to environmental change (Elmqvist et al., 2003), yet it has been little studied within the context of species interaction networks. 
Recently, response diversity of native bee communities has been identified as an important factor in maintaining watermelon pollination rates (Winfree and Kremen, 2009). In addition, for a given network size (number of species), increasing connectance (the number of links) means increased generalism of the species involved. This may provide a buffer in the responses of predators to fluctuations in prey species abundances, and generalist predators that can maintain their populations on alternative prey may be more effective biological control agents (Landis et al., 2000). However, when comparing networks of different sizes, the probability of secondary extinction (Solé and Montoya, 2001; Dunne et al., 2002a,b; Memmott et al., 2004; Rezende et al., 2007) is more likely to be related to the actual average number of interacting partners of each species (i.e. generality or link density), than a proportional measure such as connectance.

\subsubsection{How is it measured?}

The simplest measure of interaction diversity, interaction richness, is simply the number of links within a network. However, interaction richness can also be divided by the number of species in the network to calculate link density, or divided by the number of potential links (number of consumer species multiplied by the number of resource species) to calculate connectance. These binary measures also have quantitative, abundance-weighted counterparts based on information theory that incorporate the frequency and the presence of interactions (Bersier et al., 2002).

\subsubsection{How does it change with perturbation?}

Empirical studies have not yielded consistent effects. Interaction diversity of parasitoid-host food webs can be positively or negatively affected by land use intensification. Restored areas have been found to have higher interaction richness than intensively managed meadows (Albrecht et al., 2007), whereas a previous study found higher interaction richness in more intensive management systems (Tylianakis et al., 2007). The strong dependence of interaction diversity on sample sizes (Banašek-Richter et al., 2004; Tylianakis et al., 2007) may be the underlying cause of these patterns, as both studies found the highest interaction diversity in habitats with the highest insect abundances. Parasitoid-host webs in organic farms have been found to have slightly lower connectance than conventional farms, likely due to higher species richness, and these differences were not significant when quantitative network metrics were used (Macfadyen et al., 2009). Integration of exotic species into a plant pollinator network was found to have no effect on overall network connectance, although connectance among native species declined (Aizen et al., 2008). Similarly, neither connectance, link density or interaction diversity in plant-herbivore-parasitoid webs were found to be affected by invasion (Heleno et al., 2009).

\subsection{Nestedness}

\subsubsection{What is it?}

The interactions in a network are said to be nested when the species interacting with specialists are a proper subset of the species interacting with generalists. This means that as specialists are lost from the network, the core of interacting generalists remains unaltered, analogous to the peeling of layers from an onion.

\subsubsection{Why is it important?}

Specialist species are usually the first to go extinct from a network (Henle et al., 2004), but if the network is nested, the remaining species will still have others with which to interact. In the case of mutualist networks, obviously both interacting partners require the interaction. If a specialist pollinator or seed disperser goes extinct from a nested network, the plant species that it interacted with can still be pollinated/dispersed by other more generalist species (Memmott et al., 2004; Bascompte et al., 2006; Fortuna and Bascompte, 2006; Okuyama and Holland, 2008). Thus, a nested interaction structure provides a buffer against secondary extinctions or temporal fluctuations in the abundance of specialist pollinators, assuming that pollination by a generalist is approximately functionally equivalent to pollination by a specialist. In contrast, the extinction of a predator or herbivore would not cause secondary extinction of its prey or host plant species, so the extent to which nestedness may provide stability in antagonistic networks remains unclear. Furthermore, the continual arms race between plants and herbivores through defence chemicals may drive specialisation, and herbivore networks have been shown to be more specialised than mutualist networks, both phylogenetically and in terms of the number of interacting partners per species (Fontaine et al., 2009). Therefore, although the majority of research on network nestedness has centred around mutualistic interactions such as pollination and seed dispersal, the prevalence and consequences of nestedness in antagonistic networks may differ. In fact, antagonistic networks (such as predator-prey or plant-herbivore food webs) may be more compartmentalised (Lewinsohn et al., 2006; but see Thébault and Fontaine (2008)), though how this affects food web functional stability remains unclear. Nestedness and compartmentalisation need not be mutually exclusive, as interactions within a compartment may be nested (Lewinsohn et al., 2006).

\subsubsection{How is it measured?}

Usually nestedness is measured through appropriate software such as the Nestedness Temperature Calculator (NTC; Atmar and Patterson, 1995). This re-arranges the matrix of consumer (columns) by resource (rows) interactions to maximise nestedness, calculates an isocline of perfect nestedness, and then weights all the unexpected absences and unexpected presences in the interaction matrix. A more recently developed software, ANINHADO (Guimaraes and Guimaraes, 2006), adds the possibility of choosing among several null models in order to explore whether a network has significantly greater (or lower) nestedness than if species interacted randomly. Other software giving alternative measures of nestedness is provided by Rodríguez-Gironés and Santamaria (2006) BINMATNEST, which claims to improve on NTC by producing a unique isocline of perfect order and using genetic algorithms to re-arrange the matrix so it always maximises nestedness. It also implements several null models. Similarly, Almeida-Neto et al. (2008) proposed a new metric based on two properties of nestedness, namely whether marginal totals differ among rows and/or among columns, and whether the presences in less filled rows and columns coincide with the presences in more filled rows and columns. An equivalent measure of nestedness based on the degree of overlap in interacting partners across pairs of species (equivalent to the second property of the previous measure) has been independently provided by Bastolla et al. (2009). Other measures of nestedness are reviewed by Almeida-Neto et al. (2008).

\subsubsection{How does it change with perturbation?}

Super-generalist invaders become central nodes (most connected species) in the core of the nested matrix and may increase the overall value of nestedness of the network (Aizen et al., 2008; Bartomeus et al., 2008; Bjerknes et al., 2007).

\subsection{Distributions and patterns of interaction strength}

\subsubsection{What are they?}

Patterns in the strength of interactions can be important at two scales. First, at a coarse scale, there can be differences in the relative abundance of weak vs. strong interactions within the web (i.e. 
differences in the frequency distribution of interaction strengths). Several studies have now shown that networks of ecological interactions are composed of a few strong interactions within a matrix of weak interactions (Paine, 1980; Goldwasser and Roughgarden, 1997; Wootton, 1997; Bascompte et al., 2005, 2006). Second, given a certain frequency distribution, there may be differences in the way these interaction strengths are combined in species pairs; that is, differences in the interaction symmetry (Neutel et al., 2002; Bascompte et al., 2005, 2006).

\subsubsection{Why are they important?}

Although empirical evaluations are lacking, theoretical work suggests that the presence of many weak links within a network is stabilizing because they can dampen strong oscillatory consumer-resource interactions by reducing resource growth rates and consumer attack rates (McCann et al., 1998). Weak interactions may become strong at different times/locations, providing robustness under fluctuating conditions (temporal stability). In contrast, strong links make species more affected by changes in population densities of the species they are linked to; drastic changes in one species destabilise the system by spreading along the strong links (May, 1973; McCann et al., 1998; Kokkoris et al., 1999). In terms of the patterning of interaction strengths, minimising strong interactions in long loops stabilises the food web (Neutel et al., 2002). Likewise, the infrequent occurrence of two consecutive strong interactions in a food chain reduces the likelihood of a trophic cascade (Bascompte et al., 2005). Finally, Bascompte et al. (2006) found that if one partner in a plant-animal pairwise interaction had a strong mutualistic dependence, the other partner's dependence on that species was much weaker than expected by chance (i.e. pairwise interactions are asymmetric). These weak and asymmetric dependences in mutualistic networks make the entire ensemble more resistant to secondary extinctions (Bascompte et al., 2006), although as with nestedness, the importance of asymmetric dependence in antagonistic networks is unclear. Nestedness is somewhat related to interaction asymmetries. Specifically, nestedness implies asymmetric specialisation (specialists interact with generalists), that is, asymmetry at the level of the number of interactions per species. Conversely, asymmetry in dependence values is defined from pairwise interaction strengths in quantitative networks. To some extent, dependence asymmetry is a confirmation of specialisation asymmetry: if a species interacts only with a generalist species, its dependence on that is total, while the generalist may depend on a series of other species. An additional fraction of the variance in asymmetry in pairwise dependence remains, beyond that which can be explained by the level of nestedness, and therefore dependence asymmetry cannot be reduced to being identical to nestedness. Note, however, that nestedness implies asymmetric specialisation but also symmetric generalisation (both generalist plants and generalist animals interact among themselves). Therefore, this core of generalists cannot be explained based merely on an asymmetric pattern of specialisation or an asymmetric pattern of pairwise dependences. Future work should quantify the percent of variability on these measures which is independent from the variability in others.

\subsubsection{How are they measured?}

Frequency distributions can simply be plotted, or incorporated in metrics such as interaction evenness (Tylianakis et al., 2007). Patterning of interaction strengths can be estimated by calculating the probability of two given classes of interaction strength occurring in a specific combination, comparing the observed probability with that expected in an appropriate randomization (Bascompte et al., 2006).
3.3.4. How do they change with perturbation?

Theoretical work has shown that as a community assembles through waves of species invasions, the average interaction strength decreases. Communities that are not vulnerable to further species invasions tend to be those that have average interspecific interactions that are weaker than the average in the species pool (Kokkoris et al., 1999). Interestingly, this result matches several empirical studies. For example, the introduction of invasive species to a mutualist network has been shown to reduce the average dependence of each plant on each pollinator and vice versa (i.e. network-scale mutualism strength) (Aizen et al., 2008). With greater levels of invasion, invaders engaged disproportionately in the most asymmetric interactions. As interaction asymmetries can increase network stability (Bascompte et al., 2006), invaderdominated webs may be more stable and thus more difficult to restore to their previous uninvaded state (Tylianakis, 2008). Similarly, interaction evenness of parasitoid-host networks has been shown to decline in more disturbed habitats (Albrecht et al., 2007; Tylianakis et al., 2007), but interaction evenness in plant-herbivore-parasitoid multitrophic webs may not be affected by invasion (Heleno et al., 2009).

\subsection{Compartmentalisation/modularity}

\subsubsection{What is it?}

Compartments (modules) are subsets of an interaction network in which species interact frequently with one another, but little with other species outside of the compartment (May, 1973; McNaughton, 1978; Pimm, 1979; Krause et al., 2003; Rezende et al., 2009). It is not clear whether compartmentalisation and nestedness are related (networks can be highly nested within compartments, yet highly compartmentalised; Lewinsohn et al., 2006; Olesen et al., 2007). Compartmentalisation may be caused by coevolution (trophic specialisation; Olesen et al., 2007; Dupont and Olesen, 2009), such as long-tongued/billed pollinators and tubular flowers, though it may be more common in antagonistic than mutualistic networks (Lewinsohn et al., 2006; Olesen et al., 2007; see Fontaine et al. (2009) for a discussion of the evolutionary reasons behind this pattern).

\subsubsection{Why is it important?}

It is often assumed that compartmentalisation increases stability of interaction networks (May, 1973; McNaughton, 1978; Pimm, 1979; Krause et al., 2003), as disturbances will on average spread more slowly through a compartmentalised web (though they may spread quickly within a compartment). Lower trophic level resource species are more likely to be compartmentalised across habitats, with mobile consumers linking these species (Rooney et al., 2008). This highlights the importance of generalists for "gluing" individuals within modules and keeping modules together in a network. Among these generalists, highly connected species within a compartment are referred to as "module hubs" and species that link two or more compartments together are called "connectors" (Guimerà and Amaral, 2005; Olesen et al., 2007). In terms of conservation importance, extinction of module hubs or connectors is predicted to lead to the compartment or entire network (respectively) being fragmented, with cascading extinctions within or across compartments (Olesen et al., 2007). However, research on the importance of compartmentalisation for real-world network stability is lacking, and many predictions remain to be tested.

\subsubsection{How is it measured?}

A variety of algorithms exist for assessing compartmentalisation. For example, a module-finding algorithm combined with a simulated annealing optimisation approach (Guimerà and Amaral, 2005) uses a heuristic procedure to find an optimal solution to 
maximise a function called modularity. Modularity is higher if there is a strong tendency for the species of a compartment to interact among themselves, while they hardly interact with species belonging to other modules. This and similar module-finding algorithms are reviewed in the supplementary information to Olesen et al. (2007). In addition, a simple quantitative measure of compartment diversity (based on the exponential form of the Shannon index) can be used (Lewis et al., 2002).

\subsubsection{How does it change with perturbation?}

The effects of perturbations on compartmentalisation are not well studied. Empirical tests of the effects of habitat modification on quantitative compartment diversity (Lewis et al., 2002) within parasitoid-host webs have found either no effect across different habitats (Tylianakis et al., 2007) or higher compartmentalisation in restored than disturbed meadows (Albrecht et al., 2007). Highly connected generalist species may cause fusion of compartments (Olesen et al., 2007), suggesting that 'super-generalist' invasive species (Aizen et al., 2008) may increase overall network connectivity. This could potentially increase the spread of perturbations across compartments, even though the increased connectivity may reduce secondary extinctions and high interaction asymmetries in invaded webs may increase functional stability (see Sections 3.1 and 3.3 above). As a general rule, the effect of individual species extinctions or invasions will depend on the role of the species as a module hub or connector, with loss of module hubs increasing the likelihood of secondary extinctions within modules (i.e. reduces web stability), and loss of connectors decreasing the likelihood of trophic cascades across modules (i.e. increases web stability).

\subsection{Connectivity distribution}

\subsubsection{What is it?}

Connectivity is the number of interactions involving each species, which is also referred to as the 'degree' in network literature. The connectivity distribution is the frequency distribution of the number of interactions per species. This is analogous to a species abundance distribution, but instead of the abundance of each species, the number of links is plotted.

\subsubsection{Why is it important?}

Knowing a network's connectivity distribution is important for two reasons. First, it can suggest processes of network formation. For example, the random model of graph formation of Erdös and Rényi (1959), whereby a new node entering the network tends to interact with any existing nodes with the same probability, leads to an exponential connectivity distribution. On the other hand, the preferential attachment model where new nodes tend to interact preferentially with already well-connected nodes (a type of rich-gets-richer process) leads to power-law distributions. Second, the pioneering work of Albert and Barabasi (2000) demonstrated that there is a well-defined relationship between the shape of the connectivity distribution and the robustness of the network to secondary extinctions following the extinction of one species selected at random, or following a ranking from the most connected to the least connected species. Thus, while scale-free networks (those whose degree distribution follows a power-law implying that there is no single scale in connectivities but a broad continuum from specialists to super-generalists) are much more robust to random node elimination than exponential ones, they are very fragile to the deletion of the most connected nodes (Albert and Barabasi, 2000).

\subsubsection{How is it measured?}

Normally, to smooth the connectivity distributions and to make them comparable, the cumulative degree distribution (i.e. the probability of one species interacting with one or more species, with two or more species, and so on) is plotted (see Figs. 1 and 2). Then, the best model describing such connectivity distributions is fitted. In most cases this best model tends to be either an exponential distribution (representing a random network), a power-law distribution (or scale-free, depicting a complex network such as those formed through the preferential attachment mechanism) or a truncated power-law. The large majority of mutualistic networks are best described by the truncated power-law functions (Jordano et al., 2003), while the majority of food webs are best described by exponential functions (Dunne et al., 2002b).

\subsubsection{How does it change with perturbation?}

There are neither empirical assessments nor theoretical explorations of the effect of perturbations on the shape of the connectivity distribution. A tendency towards a more homogeneous distribution is found as one decreases sampling effort (Fig. 1), which may be used as a suggestion that perturbations causing loss of species or interactions will lead to a more even distribution.

\section{Stability: is it always desirable?}

Despite many potentially beneficial interpretations, stability may also be associated with system states that are not considered desirable. For example, weaker mutualism strength observed in
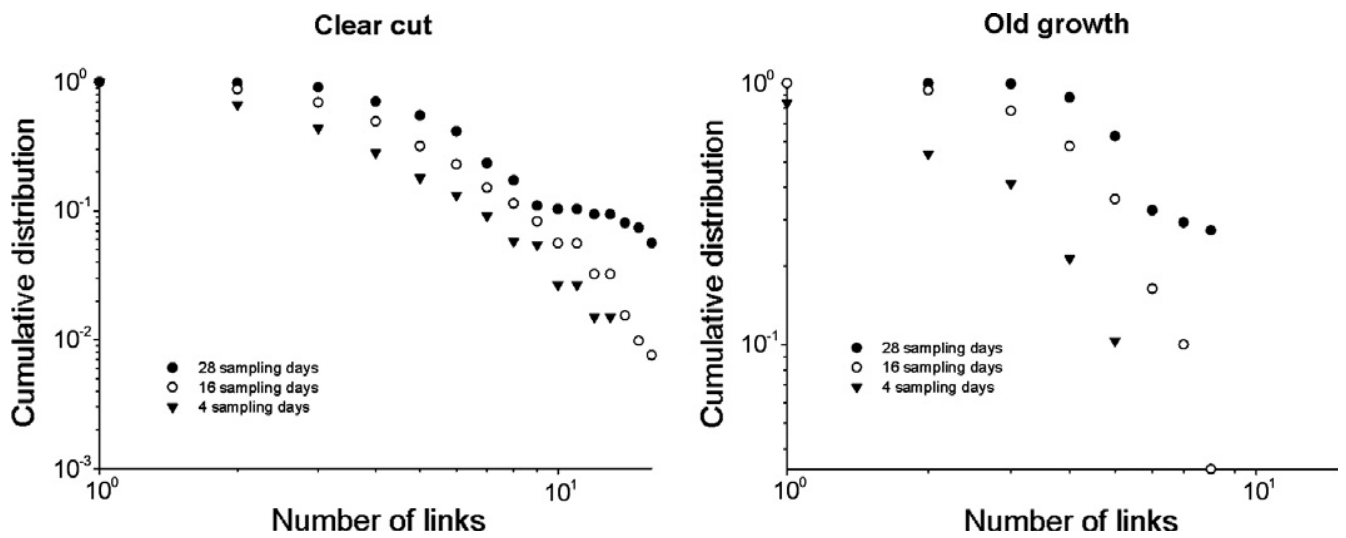

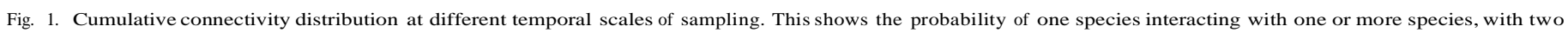

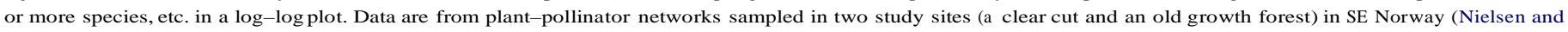
Bascompte, 2007). The sampling was carried out throughout the summer of 2004. 


\section{Lower}

Interaction richness / connectance
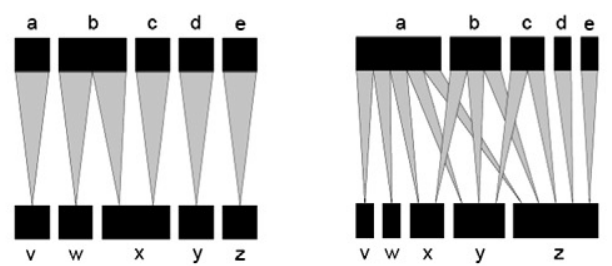

Nestedness
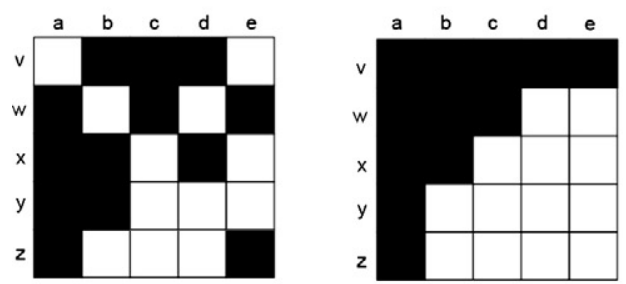

Compartmentalisation
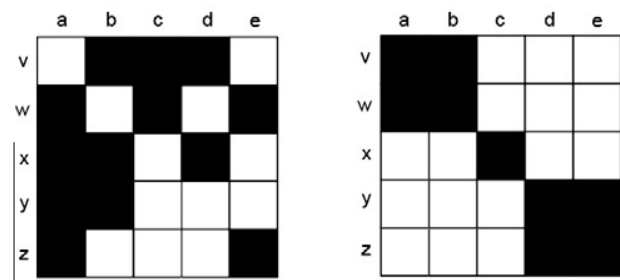

Number of weak links
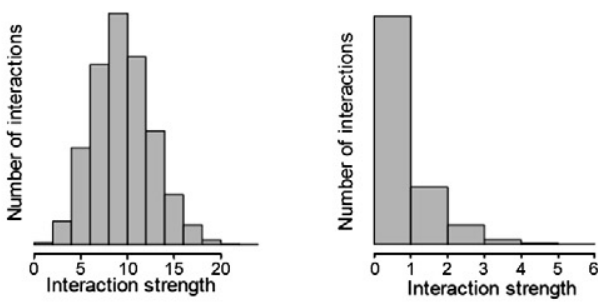

\section{Exponential}

Connectivity distribution

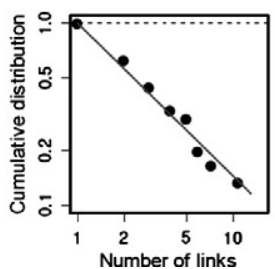

Why important?

1) Increased function

2) Greater stability of function
1) Mutualistic networks:
Buffer against secondary extinctions

2) Mutualistic networks:

Buffer against temporal fluctuations

in specialist pollinators

\section{3) Antagonistic networks:}

Role yet unclear
1) Greater stability of populations

2) Slower spread of disturbance

3) Smaller likelihood of trophic cascades
1) Greater stability of populations

2) Smaller likelihood of trophic cascades

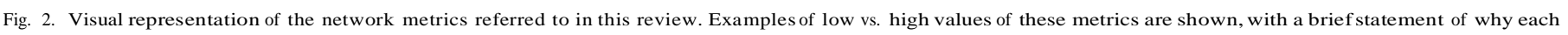
metric is important for conservation. Letters indicate consumer (a, b, c, d, e) and resource (v, w, x, y, z) species.

exotic-dominated plant-pollinator networks (Aizen et al., 2008) is associated with stability (see Section 3.3 above), and this may lead to self-perpetuating positive feedbacks that make invader-dominated communities less likely to switch to their previous uninvaded state. Similarly, the presence of many weak and few strong interactions is known to promote resistance to external perturbations (McCann et al., 1998). Recent studies have shown that para- sitoid-host food webs from highly modified habitats have few strong (highly frequent) interactions and many weak interactions (i.e. a decrease in the evenness of interaction strengths) (Albrecht et al., 2007; Tylianakis et al., 2007), characteristics that are normally associated with stability. In contrast, natural habitats tended to have numerous moderate-strength interactions (Tylianakis et al., 2007). Although it is unclear whether this will make the webs 
of modified habitats more resistant, it nevertheless raises the question of whether maintaining stability is always consistent with conservation objectives.

\section{Impediments to implementing conservation of network} structure

The first step required for the conservation of interaction network structure is to determine the most important structural elements, as outlined above. Yet, even if we settle on a useful measure of the most important structural attributes that should be conserved, how could this be put into practice? For conservation of network structure to ever be implemented widely, two requirements must be met. First, it must be demonstrated that information on network structure provides significant advantages over current conservation/monitoring foci (e.g. species diversity). We hope that from the above discussion, it will be clear that network structure may have important implications for ecosystem stability and functioning, and its conservation is thus warranted. Second, the hurdle represented by practical constraints may be greater than the theoretical challenges, and the integration of network structure into conservation monitoring may face resistance if it requires significantly more effort and resources than are currently employed in conserving species.

Fundamental to these practical constraints is the difficulty of observing interactions (particularly across systems), compared with simply counting species. Ease of recording interactions between certain organisms such as host insects and their parasitoids, or plants and their flower visitors, has almost certainly caused the over-representation of these network types in the literature. The paucity of quantitative food webs involving generalist predators may improve as DNA techniques and libraries improve our ability to quantify feeding links through gut content analyses (JuradoRivera et al., 2009). In the meantime, we must continue to use easily recorded kinds of interactions, or where interactions are logistically difficult to measure, we must rely on network metrics that require as few observations as possible to yield a representative view of the whole network.
A

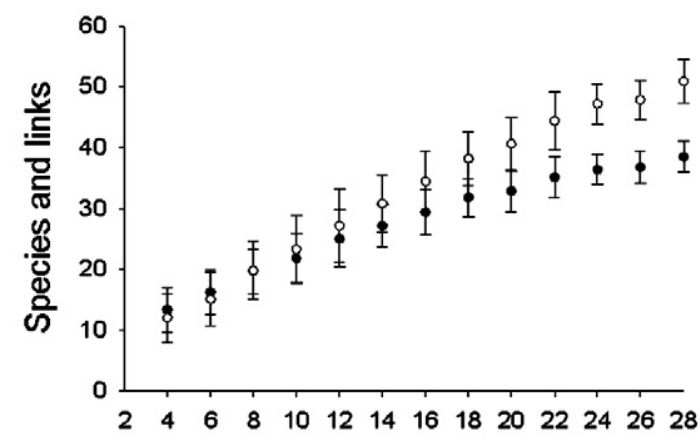

C
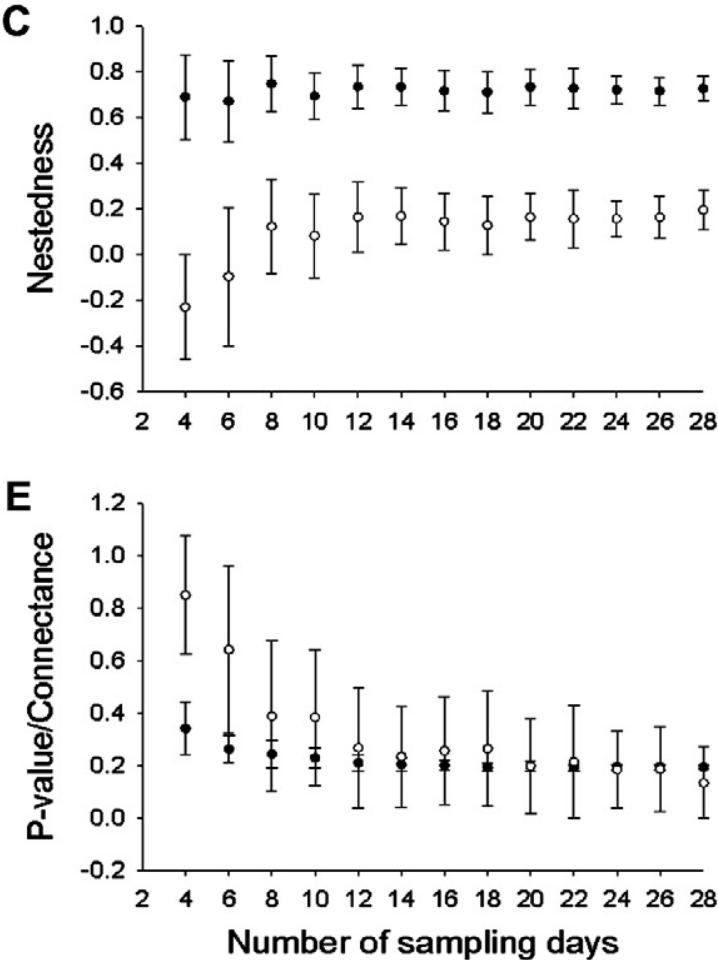

B

Old growth forest

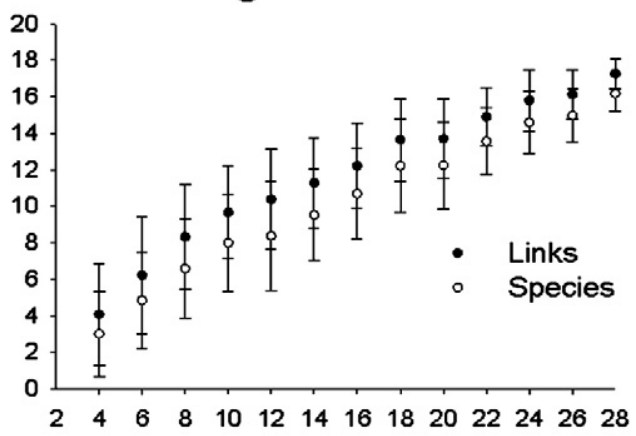

D 1

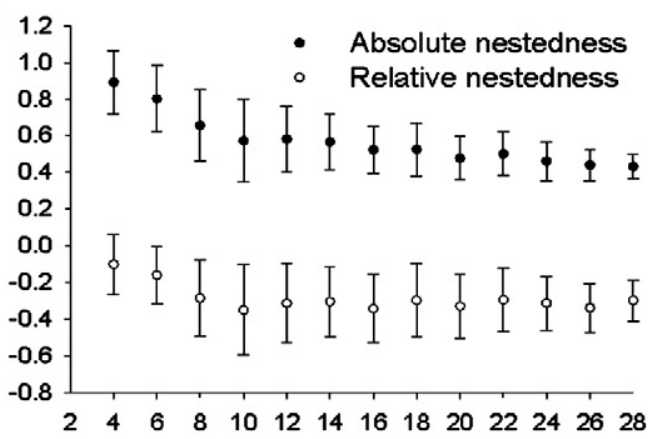

F 1

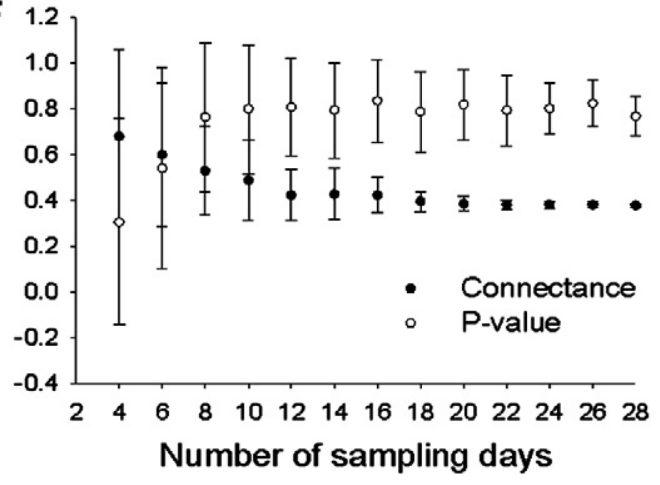

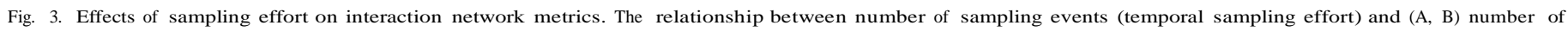

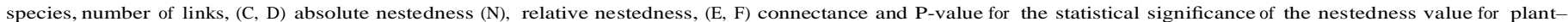

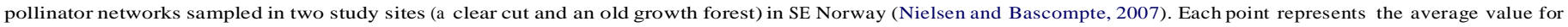
the measure, obtained from 50 random combinations of $4,6,8, \ldots$ up to 28 sampling days. Error bars are standard deviations. 
Simple measures of interaction diversity (above) could potentially be used to guide conservation efforts, but these have some drawbacks. First, some of these metrics (e.g. link density, interaction richness) are notoriously sensitive to the number of species in the network and to sampling effort (Goldwasser and Roughgarden, 1997; Bersier et al., 1999; Nielsen and Bascompte, 2007). Thus, the slow rate of accumulation (saturation) of interactions compared with species as sampling scale increases (Fig. 3A and B) may impede the incorporation of interaction diversity into conservation, even if it were highly meaningful. Connectance asymptotes more quickly than species richness with increased sampling effort (Nielsen and Bascompte, 2007; compare Fig. 3A and B with Fig. 3E and F), making it potentially more useful as an index of network structure than interaction richness. Absolute and relative nestedness and the significance value of nestedness appear to asymptote quicker than species diversity (compare Fig. $3 \mathrm{~A}$ and $\mathrm{B}$ with Fig. $3 \mathrm{C}$ and $\mathrm{D}$ ), and cumulative connectivity distribution may shift from an exponential curve to a power-law with increased sampling effort (Fig. 1). These effects of sampling effort on network metrics may largely determine their usefulness in conservation monitoring, as those attributes that are most sensitive to sampling will be most difficult to monitor effectively.

\section{Conclusions}

There are clearly a number of important emergent properties of stability and function that are conferred by interaction network structure. Nevertheless, the difficulty in accurately measuring some of these metrics is a potential drawback, and a choice must be made between the information that can be gained from a given metric and its ease of measurement in practice.

An additional problem is that commonly-used network metrics do not incorporate the identity of interactions (although this does not apply to nestedness; see also Laliberté and Tylianakis (in press)) or traits of interactors. If conserving diversity of interactions per se is the goal, existing metrics could suffice. However, conservation biologists recognise that species composition and preservation of native species is a greater priority than conservation of species richness itself. Similarly, unless interactions between species are deemed to be interchangeable, characterisation of networks based on simple metrics may not yield results that are always useful to conservation. This, combined with the practical hurdle of the sampling effort required to catalogue large numbers of interactions, suggests that it may be preferable to focus our attention on particular characteristics of interaction networks that confer functional outcomes, rather than interaction diversity or complexity per se. An alternative solution for quantifying changes to interaction networks is to directly analyse through multivariate analyses (e.g. Legendre and Anderson, 1999) the most fundamental units of an interaction network: the composition and relative frequency of its constituent interactions (Vázquez and Simberloff, 2003; Laliberté and Tylianakis, in press), rather than deriving simple metrics from network matrices.

Unlike interaction diversity, nestedness does not seem to change considerably with increasing sampling effort (Fig. 3C and D). This, combined with its importance for reducing secondary extinctions (Memmott et al., 2004), suggests that nestedness could be a good candidate for monitoring changes in the structure of mutualist networks. Connectance (or link density when different sized networks are compared) may also be a useful measure of network structure due to its ease of computation, rapid saturation with increased sampling effort, and potential link to functional redundancy of interactions (resilience). Connectivity distribution is related to nestedness and is also a good descriptor of network robustness which has been widely used in other fields. This mea- sure provides a clear link between network structure and robustness and identifies the 'glue' keeping the network together - that is, the hubs or super-generalist species in some heterogeneous ecological networks. It illustrates how robustness greatly depends on perturbation type, as heterogeneous networks are very robust to random extinctions and yet are very fragile when hub species go extinct. That said, connectivity distribution may be influenced by sampling effort as illustrated in Fig. 1 (skewed distributions may seem less heterogeneous if sampling effort is lower).

In conclusion, the incorporation of network structure into conservation monitoring and strategy is challenging yet promising, especially for those metrics that saturate more quickly with sampling effort than species diversity. Rather than focusing on any single metric, conservation of complex emergent properties such as network stability will likely require monitoring of a suite of metrics. This could include: (1) some measure or measures of connectivity (e.g. connectance, link density, average generality) and its distribution (which may affect the likelihood of secondary extinction), (2) compartmentalisation (particularly when the spread of pollutants or perturbations threatens species), and (3) nestedness, when stability of functions such as pollination is of concern. However, further research on the functional consequences of network structure, and the response of different metrics to perturbation, will be required before a truly convincing argument for the conservation of network structure can be made.

\section{Acknowledgements}

R.K. Didham and two anonymous reviewers provided helpful comments on an earlier draft of this manuscript. J.M.T. acknowledges support from the Marsden Fund (UOC-0705 and UOC0802), E.L. is supported by the University of Canterbury, Education New Zealand, and FQRNT, Canada, A.N. is supported by The Research Council of Norway (project 154442/720), and J.B. is supported by the European Heads of Research Councils, the European Science Foundation, and the EC Sixth Framework Programme through a European Young Investigator (EURYI) Award.

\section{References}

Aizen, M., Morales, C., Morales, J., 2008. Invasive mutualists erode native pollination webs. PLoS Biology 6 (2), e31.

Albert, R., Barabasi, A.L., 2000. Topology of evolving networks: local events and universality. Physical Review Letters 85, 5234-5237.

Albrecht, M., Duelli, P., Schmid, B., Muller, C.B., 2007. Interaction diversity within quantified insect food webs in restored and adjacent intensively managed meadows. Journal of Animal Ecology 76, 1015-1025.

Almeida-Neto, M., Guimaraes, P., Guimaraes, P.R., Loyola, R.D., Ulrich, W., 2008. A consistent metric for nestedness analysis in ecological systems: reconciling concept and measurement. Oikos 117, 1227-1239.

Armsworth, P.R., Chan, K.M.A., Daily, G.C., Ehrlich, P.R., Kremen, C., Ricketts, T.H., Sanjayan, M.A., 2007. Ecosystem-service science and the way forward for conservation. Conservation Biology 21, 1383-1384.

Atmar, W., Patterson, B.D., 1995. The Nestedness Temperature Calculator: A Visual Basic Program, Including 294 Presence-Absence Matrices. AICS Research, Univ. Park, NM and Field Museum, Chicago. <http://www.aicsresearch.com/ nestedness/tempcalc.html>.

Banašek-Richter, C., Cattin, M.F., Bersier, L.F., 2004. Sampling effects and the robustness of quantitative and qualitative food-web descriptors. Journal of Theoretical Biology 226, 23-32.

Bartomeus, I., Vila, M., Santamaría, L., 2008. Contrasting effects of invasiveplants in plant-pollinator network. Oecologia 155, 761-770.

Bascompte, J., Jordano, P., 2007. Plant-animal mutualistic networks: the architecture of biodiversity. Annual Review of Ecology, Evolution and Systematics 38, 567-593.

Bascompte, J., Melian, C.J., Sala, E., 2005. Interaction strength combinations and the overfishing of a marine food web. Proceedings of the National Academy of Sciences of the United States of America 102, 5443-5447.

Bascompte, J., Jordano, P., Olesen, J.M., 2006. Asymmetric coevolutionary networks facilitate biodiversity maintenance. Science 312, 431-433.

Bastolla, U., Fortuna, M.A., Pascual-Garcia, A., Ferrera, A., Luque, B., Bascompte, J., 2009. The architecture of mutualistic networks minimizes competition and increases biodiversity. Nature 458, 1018-1020. 
Bersier, L.F., Dixon, P., Sugihara, G., 1999. Scale-invariant or scale-dependent behavior of the link density property in food webs: a matter of sampling effort? American Naturalist 153, 676-682.

Bersier, L.F., Banasek-Richter, C., Cattin, M.F., 2002. Quantitative descriptors of foodweb matrices. Ecology 83, 2394-2407.

Bjerknes, A.L., Totland, O., Hegland, S.J., Nielsen, A., 2007. Do alien plant invasions really affect pollination success in native plant species? Biological Conservation $138,1-12$.

Callicott, J.B., Crowder, L.B., Mumford, K., 1999. Current normative concepts in conservation. Conservation Biology 13, 22-35.

Carvalheiro, L.G., Barbosa, E.R.M., Memmott, O.J., 2008. Pollinator networks, alien species and the conservation of rare plants: Trinia glauca as a case study. Journal of Applied Ecology 45, 1419-1427.

Díaz, S., Fargione, J., Chapin, F.S., Tilman, D., 2006. Biodiversity loss threatens human well-being. PLoS Biology 4, 1300-1305.

Dobson, A., Lodge, D., Alder, J., Cumming, G.S., Keymer, J., McGlade, J., Mooney, H., Rusak, J.A., Sala, O., Wolters, V., Wall, D., Winfree, R., Xenopoulos, M.A., 2006. Habitat loss, trophic collapse and the decline of ecosystem services. Ecology 87, 1915.

Dunne, J.A., Williams, R.J., 2009. Cascading extinctions and community collapse in model food webs. Philosophical Transactions of the Royal Society B - Biological Sciences 364, 1711-1723.

Dunne, J.A., Williams, R.J., Martinez, N.D., 2002a. Network structure and biodiversity loss in food webs: robustness increases with connectance. Ecology Letters 5, $558-5567$.

Dunne, J., Williams, R., Martinez, N., 2002b. Food-web structure and network theory: the role of connectance and size. Proceedings of the National Academy of Sciences of the United States of America 99, 12917-12922.

Dupont, Y.L., Olesen, J.M., 2009. Ecological modules and roles of species in heathland plant-insect flower visitor networks. Journal of Animal Ecology 78, 346-353.

Ehrlich, P., Walker, B., 1998. Rivets and redundancy. BioScience 48, 387.

Elmqvist, T., Folke, C., Nystrom, M., Peterson, G., Bengtsson, J., Walker, B., Norberg, J., 2003. Response diversity, ecosystem change, and resilience. Frontiers in Ecology and the Environment 1, 488-494.

Erdös, P., Rényi, A., 1959. On random graphs. Publicationes Mathematicae 6, 290297.

Finke, D.L., Denno, R.F., 2004. Predator diversity dampens trophic cascades. Nature 429, 407-410.

Fontaine, C., Dajoz, I., Meriguet, J., Loreau, M., 2006. Functional diversity of plantpollinator interaction webs enhances the persistence of plant communities. PLoS Biology 4, 129-135.

Fontaine, C., Thébault, E., Dajoz, I., 2009. Are insect pollinators more generalist than insect herbivores? Proceedings of the Royal Society B - Biological Sciences 276, 3027-3033.

Fortuna, M.A., Bascompte, J., 2006. Habitat loss and the structure of plant-animal mutualistic networks. Ecology Letters 9, 278-283.

Goldwasser, L., Roughgarden, J., 1997. Sampling effects and the estimation of foodweb properties. Ecology 78, 41-54.

Grimm, V., Wissel, C., 1997. Babel, or the ecological stability discussions: an inventory and analysis of terminology and a guide for avoiding confusion. Oecologia 109, 323-334.

Guimaraes, P.R., Guimaraes, P., 2006. Improving the analyses of nestedness for large sets of matrices. Environmental Modelling \& Software 21, 1512-1513.

Guimerà, R., Amaral, L.A.N., 2005. Functional cartography of complex metabolic networks. Nature 433, 895-900.

Hegland, S.J., Nielsen, A., Lázaro, A., Bjerknes, A.-L., Totland, Ø., 2009. How does climate warming affect plant-pollinator interactions? Ecology Letters 12, 184195

Heleno, R.H., Ceia, R.S., Ramos, J.A., Memmott, J., 2009. Effects of alien plants on insect abundance and biomass: a food-web approach. Conservation Biology 23 , 410-419.

Henle, K., Davies, K.F., Kleyer, M., Margules, C., Settele, J., 2004. Predictors of species sensitivity to fragmentation. Biodiversity and Conservation 13, 207251.

Hoehn, P., Tscharntke, T., Tylianakis, J.M., Steffan-Dewenter, I., 2008. Functional group diversity of bee pollinators increases crop yield. Proceedings of the Royal Society B - Biological Sciences 275, 2283-2291.

Ives, A.R., Carpenter, S.R., 2007. Stability and diversity of ecosystems. Science 317, 58-62.

Jordano, P., Bascompte, J., Olesen, J.M., 2003. Invariant properties in coevolutionary networks of plant-animal interactions. Ecology Letters 6, 69-81.

Jurado-Rivera, J.A., Vogler, A.P., Reid, C.A.M., Petitpierre, E., Gomez-Zurita, J., 2009. DNA barcoding insect-host plant associations. Proceedings of the Royal Society B - Biological Sciences 276, 639-648.

Kareiva, P., Watts, S., McDonald, R., Boucher, T., 2007. Domesticated nature: shaping landscapes and ecosystems for human welfare. Science 316, 1866-1869.

Kokkoris, G.D., Troumbis, A.Y., Lawton, J.H., 1999. Patterns of species interaction strength in assembled theoretical communities. Ecology Letters 2, 70-74.

Krause, A.E., Frank, K.A., Mason, D.M., Ulanowicz, R.E., Taylor, W.W., 2003. Compartments revealed in food-web structure. Nature 426, 282-285.

Laliberté, E. Tylianakis, J.M., in press. Deforestation homogenizes tropical parasitoid-host networks. Ecology.

Landis, D.A., Wratten, S.D., Gurr, G.M., 2000. Habitat management to conserve natural enemies of arthropod pests in agriculture. Annual Revue of Entomology 45, 175-201.
Lawton, J.H., Brown, V.K., 1993. Redundancy in ecosystems. In: Schulze, E.-D., Mooney, H.A. (Eds.), Biodiversity and Ecosystem Function. Springer-Verlag, Berlin, pp. 255-270.

Legendre, P., Anderson, M.J., 1999. Distance-based redundancy analysis: testing multispecies responses in multifactorial ecological experiments. Ecological Monographs 69, 1-24.

Lewinsohn, T.M., Prado, P.I., Jordano, P., Bascompte, J., Olesen, J.M., 2006. Structure in plant-animal interaction assemblages. Oikos 113, 174-184.

Lewis, O.T., Memmott, J., Lasalle, J., Lyal, C.H.C., Whitefoord, C., Godfray, H.C.J., 2002 Structure of a diverse tropical forest insect-parasitoid community. Journal of Animal Ecology 71, 855-873.

Lopezaraiza-Mikel, M., Hayes, R., Whalley, M., Memmott, J., 2007. The impact of an alien plant on a native plant-pollinator network: an experimental approach. Ecology Letters 10, 539-550.

Luck, G.W., Harrington, R., Harrison, P.A., Kremen, C., Berry, P.M., Bugter, R., Dawson, T.P., de Bello, F., Diaz, S., Feld, C.K., Haslett, J.R., Hering, D., Kontogianni, A., Lavorel, S., Rounsevell, M., Samways, M.J., Sandin, L., Settele, J., Sykes, M.T., van den Hove, S., Vandewalle, M., Zobel, M., 2009. Quantifying the contribution of organisms to the provision of ecosystem services. BioScience 59, 223-235.

Macfadyen, S., Gibson, R., Polaszek, A., Morris, R.J., Craze, P.G., Planque, R., Symondson, W.O.C., Memmott, J., 2009. Do differences in food web structure between organic and conventional farms affect the ecosystem service of pest control? Ecology Letters 12, 229-238.

May, R., 1973. Stability and Complexity in Model Ecosystems. Princeton University Press.

McCann, K., 2007. Protecting biostructure. Nature 446, 29.

McCann, K., Hastings, A., Huxel, G.R., 1998. Weak trophic interactions and the balance of nature. Nature 395, 794-798.

McCann, K.S., Rasmussen, J.B., Umbanhowar, J., 2005. The dynamics of spatially coupled food webs. Ecology Letters 8, 513-523.

McNaughton, S.J., 1978. Stability and diversity of ecological communities. Nature 274, 251-253.

Memmott, J., Waser, N.M., Price, M.V., 2004. Tolerance of pollination networks to species extinctions. Proceedings of the Royal Society of London Series B Biological Sciences 271, 2605-2611.

Memmott, J., Craze, P.G., Waser, N.M., Price, M.V., 2007. Global warming and the disruption of plant-pollinator interactions. Ecology Letters 10, 710-717.

Millennium Ecosystem Assessment, 2005. Ecosystems and Human Well-being: Scenarios. Island Press, Washington, DC, USA.

Montoya, J.M., Rodriguez, M.A., Hawkins, B.A., 2003. Food web complexity and higher-level ecosystem services. Ecology Letters 6, 587-593.

Montoya, J.M., Pimm, S.L., Sole, R.V., 2006. Ecological networks and their fragility. Nature 442, 259-264.

Naeem, S., 1998. Species redundancy and ecosystem reliability. Conservation Biology 12, 39-45.

Neutel, A.M., Heesterbeek, J.A.P., de Ruiter, P.C., 2002. Stability in real food webs: weak links in long loops. Science 296, 1120-1123.

Nielsen, A., Bascompte, J., 2007. Ecological networks, nestedness and sampling effort. Journal of Ecology 95, 1134-1141.

Okuyama, T., Holland, J., 2008. Network structural properties mediate the stability of mutualistic communities. Ecology Letters 11, 208-216.

Olesen, J., Bascompte, J., Dupont, Y., Jordano, P., 2007. The modularity of pollination networks. Proceedings of the National Academy of Sciences of the United States of America 104, 19891-19896.

Paine, R.T., 1980. Food webs: linkage, interaction strength and community infrastructure. Journal of Animal Ecology 49, 667-685.

Pascual, M., Dunne, J.A. (Eds.), 2006. Ecological Networks: Linking Structure to Dynamics. Oxford University Press.

Pimm, S.L., 1979. Structure of food webs. Theoretical Population Biology 16, 144 158.

Post, W.M., Pimm, S.L., 1983. Community assembly and food web stability. Mathematical Biosciences 64, 169-192.

Rezende, E., Lavabre, J., Guimaraes Jr., P.R., Jordano, P., Bascompte, J., 2007. Nonrandom co-extinctions in phylogenetically structured mutualistic networks. Nature 448, 925-928.

Rezende, E.L., Albert, E.M., Fortuna, M.A., Bascompte, J., 2009. Compartments in a marine food web associated with phylogeny, body mass and habitat structure. Ecology Letters 12, 779-788.

Rodríguez-Gironés, M.A., Santamaria, L., 2006. A new algorithm to calculate the nestedness temperature of presence-absence matrices. Journal of Biogeography 33, 924-935.

Rooney, N., McCann, K.S., Moore, J.C., 2008. A landscape theory for food web architecture. Ecology Letters 11, 867-881.

Snyder, W.E., Snyder, G.B., Finke, D.L., Straub, C.S., 2006. Predator biodiversity strengthens herbivore suppression. Ecology Letters 9, 789-796.

Solé, R.V., Montoya, J.M., 2001. Complexity and fragility in ecological networks. Proceedings of the Royal Society B - Biological Sciences 268, 2039-2045.

Srinivasan, U.T., Dunne, J.A., Harte, J., Martinez, N.D., 2007. Response of complex food webs to realistic extinction sequences. Ecology 88, 671-682.

Tallis, H., Kareiva, P., Marvier, M., Chang, A., 2008. An ecosystem services framework to support both practical conservation and economic development. Proceedings of the National Academy of Sciences of the United States of America 105, 94579464.

Terborgh, J., Lopez, L., Nuñez, P.V., Rao, M., Shahabuddin, G., Orihuela, G., Riveros, M., Ascanio, R., Adler, G.H., Lambert, T.D., Balbas, L., 2001. Ecological meltdown in predator-free forest fragments. Science 294, 1923-1926. 
Thébault, E., Fontaine, C., 2008. Does asymmetric specialization differ between mutualistic and trophic networks? Oikos 117, 555-563.

Thompson, J.N., 1994. The Coevolutionary Process. University of Chicago Press, Chicago, IL.

Tylianakis, J.M., 2008. Understanding the web of life: the birds, the bees and sex with aliens. PLoS Biology 6, e47.

Tylianakis, J.M., Tscharntke, T., Lewis, O.T., 2007. Habitat modification alters the structure of tropical host-parasitoid food webs. Nature 445, 202-205.

Tylianakis, J.M., Didham, R.K., Bascompte, J., Wardle, D.A., 2008. Global change and species interactions in terrestrial ecosystems. Ecology Letters 11, 13511363.

Vázquez, D., Simberloff, D., 2003. Changes in interaction biodiversity induced by an introduced ungulate. Ecology Letters 6, 1077-1083.

Visser, M.E., Both, C., 2005. Shifts in phenology due to global climate change: the need for a yardstick. Proceedings of the Royal Society B - Biological Sciences 272, 2561-2569.
Walker, B.H., 1992. Biodiversity and ecological redundancy. Conservation Biology 6, 18-23.

Wilson, K.A., Underwood, E.C., Morrison, S.A., Klausmeyer, K.R., Murdoch, W.W., Reyers, B., Wardell-Johnson, G., Marquet, P.A., Rundel, P.W., McBride, M.F., Pressey, R.L., Bode, M., Hoekstra, J.M., Andelman, S., Looker, M., Rondinini, C., Kareiva, P., Shaw, M.R., Possingham, H.P., 2007. Conserving biodiversity efficiently: what to do, where, and when. PLoS Biology 5, 1850-1861.

Winfree, R., Kremen, C., 2009. Are ecosystem services stabilized by difference among species? A test using crop pollination. Proceedings of the Royal Society B - Biological Sciences 276, 229-237.

Wootton, J.T., 1997. Estimates and tests of per-capita interaction strength: diet, abundance, and impact of intertidally-foraging birds. Ecological Monographs 67, 45-64.

Yachi, S., Loreau, M., 1999. Biodiversity and ecosystem productivity in a fluctuating environment: the insurance hypothesis. Proceedings of the National Academy of Sciences of the United States of America 96, 1463-1468. 